\title{
Apport de L'échographie dans le Diagnostic Anténatal du Syndrome de Prune Belly : À Propos d'un Cas et Revue de la Littérature
}

\author{
Meryem Belmajdoub, \\ Hekmat Chaara, \\ Fatima Zehra, \\ Fdili Alaoui, \\ Sofia Jayi, \\ Moulay Abdelilah Melhouf, \\ Service de Gynécologie Obstétrique 2, \\ Centre Hospitalier Universitaire HASSAN 2, Fès, Maroc
}

Doi:10.19044/esj.2019.v15n36p296 URL:http://dx.doi.org/10.19044/esj.2019.v15n36p296

\section{Résumé}

L'utilisation de l'échographie au premier trimestre de la grossesse et en particulier par voie endovaginale, permet la découverte précoce de mégavessie et donc des syndromes associés. Le syndrome de Prune Belly ou syndrome d'Eagle-Barrett est une malformation complexe rare avec nette prédominance masculine. Il se caractérise par la triade : aplasie ou hypoplasie de la musculature abdominale, dilatations des voies urinaires et cryptorchidie bilatérale. Il s'associe dans $75 \%$ des cas, à des atteintes pulmonaires, squelettiques, cardiaques et gastro-intestinales. Nous rapportons le cas d'un syndrome de Prune Belly découvert à 20 semaines d'aménorrhée (SA). À travers ce cas et la lumière d'une revue de la littérature récente, nous soulignerons l'intérêt de l'échographie anténatale dans le dépistage de cette malformation ainsi que les caractéristiques diagnostiques et thérapeutiques de cette entité rare.

Mots-Clés: Syndrome Prune Belly, Diagnostic Anténatal, Mégavessie, Hypoplasie Paroi Abdominale, Cryptorchidie 


\title{
Contribution of Ultrasound in the Antenatal Diagnosis of Prune Belly Syndrome: A Case Report and a Review of the Literature
}

\author{
Meryem Belmajdoub, \\ Hekmat Chaara, \\ Fatima Zehra, \\ Fdili Alaoui, \\ Sofia Jayi, \\ Moulay Abdelilah Melhouf, \\ Service de Gynécologie Obstétrique 2, \\ Centre Hospitalier Universitaire HASSAN 2, Fès, Maroc
}

\begin{abstract}
The use of ultrasound in the first trimester of pregnancy and in particular by the endovaginal way helps in the early discovery of megascessy and associated syndromes. Prune-Belly syndrome or Eagle-Barrett syndrome is a rare complex malformation with marked male predominance. It is characterized by the triad: aplasia or hypoplasia of the abdominal musculature, dilations of the urinary tract, and bilateral cryptorchidism. It is associated in $75 \%$ of the cases with pulmonary, skeletal, cardiac, and gastro-intestinal disorders. We report the case of Belly plum syndrome discovered at 20 weeks of amenorrhea (SA). Through this case and in the light of a review of the recent literature, we will highlight the importance of antenatal ultrasound in screening for this malformation as well as the diagnostic and therapeutic features of this rare entity.
\end{abstract}

Keywords: Prune Belly Syndrome, Antenatal Diagnosis, Megavessie, Abdominal Wall Hypotonia, Cryptorchidism

\section{Introduction}

La généralisation de l'échographie au premier trimestre de la grossesse et en particulier par voie endovaginale permet la découverte précoce d'une mégavessie et donc des syndromes associés (Megan et al., 2006). Le syndrome de Prune-Belly est une malformation complexe rare, caractérisé par la triade : aplasie ou hypoplasie de la musculature abdominale, dilatations des voies urinaires et cryptorchidie bilatérale (Aaronson et al., 2001).Il s'associe dans 
$75 \%$ des cas à des atteintes pulmonaires, squelettiques, cardiaques et gastrointestinales. Il existe également des formes dites incomplètes ou partielles plus fréquentes chez le sexe féminin et qui sont connues sous le nom de Syndrome de Pseudo-Prune Belly. L'évolution de cette entité est caractérisée par plusieurs complications et le pronostic dépend surtout de la sévérité de l'atteinte rénale (Aaronson et al., 2001). Nous rapportons le cas d'un syndrome de Prune Belly découvert à 20 semaines d'aménorrhée (SA) chez une primigeste de 22 ans. À travers ce cas et une revue de la littérature récente, nous soulignerons l'intérêt de l'échographie anténatale dans le dépistage de ces malformations ainsi que les caractéristiques diagnostiques et thérapeutiques de cette entité rare.

\section{Observation}

Nous rapportons le cas d'une patiente âgée de 22 ans primigeste, sans antécédents médicaux ou chirurgicaux particuliers, sans notion de mariage consanguin, référée dans notre formation à 18SA, suite à la découverte d'une mégavessie, et dont l'échographie obstétricale a objectivé : une grossesse évolutive avec grosse vessie à parois épaissies et signe de récessus sous vésical, les deux reins sont hyperéchogènes avec des pyelons bien visibles communicants avec les calices, le diagnostic d'une valve de l'urètre postérieure a été suspecté initialement. Un contrôle échographique à 2 semaines (soit à 20SA) a objectivé :la présence d'une mégavessie énorme prenant tout l'abdomen et refoulant le diaphragme (Figure 1), une dilatation pyélo-calicielle avec conservation de la différenciation cortico-médullaire (Figure 2), une paroi abdominale qui parait très fine ou même absente (Figure 1), le liquide amniotique était en quantité diminuée, le diagnostic d'un syndrome de Prune Belly a été suspecté, une IRM fotale a été faite revenue en faveur d'une mégavessie fœtale avec dilatation urétèro-pyélo-calicielle bilatérale rentrant probablement dans le cadre d'un syndrome de Prune Belly (Figure 3). Aucune autre malformation n'a été détectée au cours du suivi de la grossesse, ainsi le diagnostic d'un syndrome de Prune Belly a été retenu. À 40 SA, la patiente a accouché par voie basse, un nouveau-né de sexe masculin, pesant $3200 \mathrm{~g}$, avec à l'examen clinique la présence d'une détresse respiratoire côte 5/10, présence d'une paroi abdominale flasque avec perception d'une masse prenant tout l'abdomen, fistule au niveau de l'ouraque ramenant des urines avec cryptorchidie bilatérale (Figure 4). Le nouveau-né a été hospitalisé initialement en réanimation néonatale avec bonne évolution hémodynamique, il a bénéficié d'une échographie abdomino-pelvienne qui a objectivé une importante urétèro-hydro-néphrose bilatérale associée à une mégavessie et une aplasie des muscles abdominaux avec une cryptorchidie, étant le tout compatible avec un syndrome de Prune Belly (Figure 5). Le nouveau-né a été vu par les chirurgiens pédiatres et il a été mis sous 
antibiothérapie avec un sondage vésicale vu que la fistule de l'ouraque ramène des urines à l'extérieur avec au bilan biologique absence d'insuffisance rénale. Au cours de la surveillance le nouveau-né a présenté deux épisodes de fièvre avec au bilan infectieux une CRP élevée avec un ECBU (Examen cytobactériologique des urines) positif à Escherichia coli pour lequel il a été mis sous antibiothérapie avec bonne évolution. Actuellement le nouveau-né est toujours sous surveillance et il sera candidat à une abdominoplastie avec orchidopexie et reconstruction des voies urinaires en fonction de son évolution.

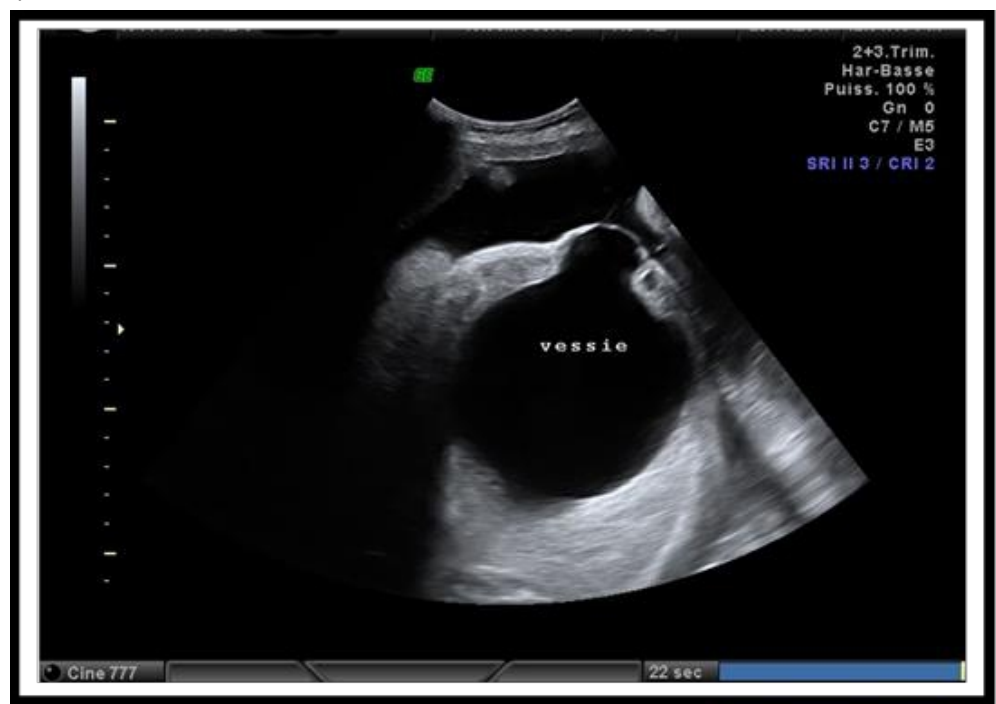

Figure 1. Echographie anténatale

Mégavessie énorme prenant tout abdomen avec paroi abdominale amincie.

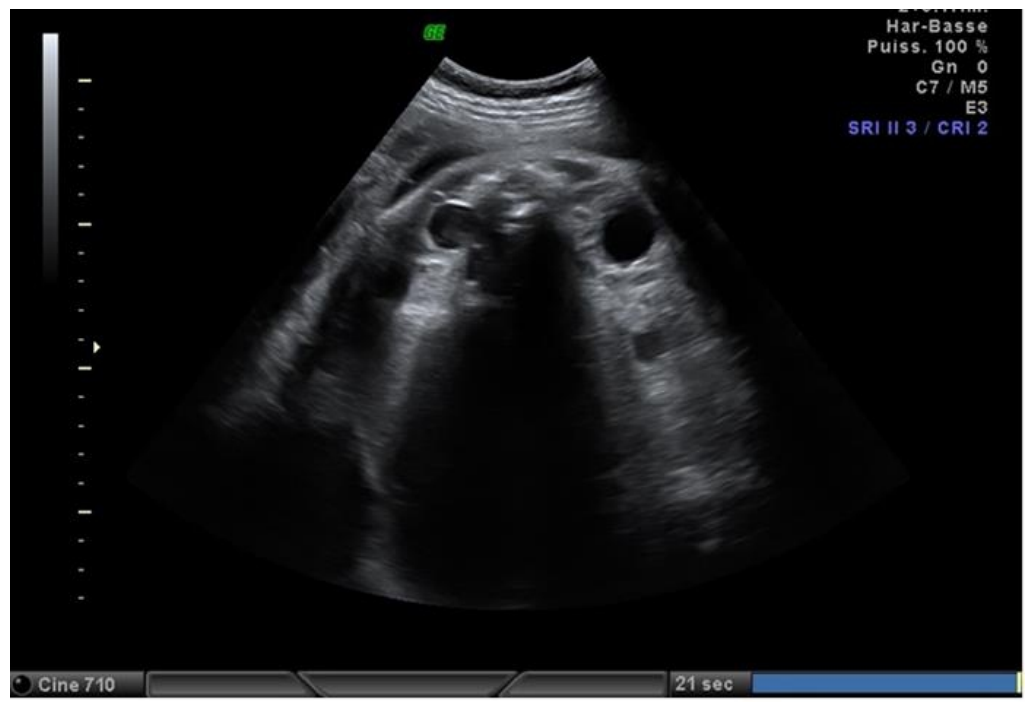

Figure 2. Echographie anténatale.

Dilatation pyélo-calicielle avec conservation de la différenciation cortico-médullaire. 


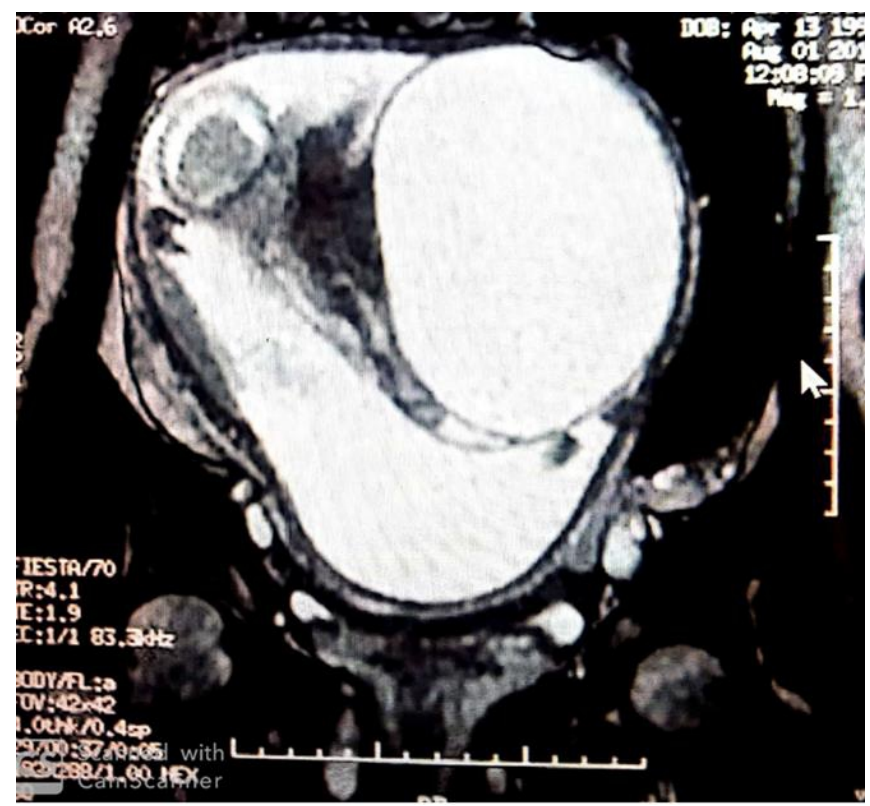

Figure 3. IRM FOETALE

Mégavessie énorme prenant tout abdomen avec paroi abdominale amincie.

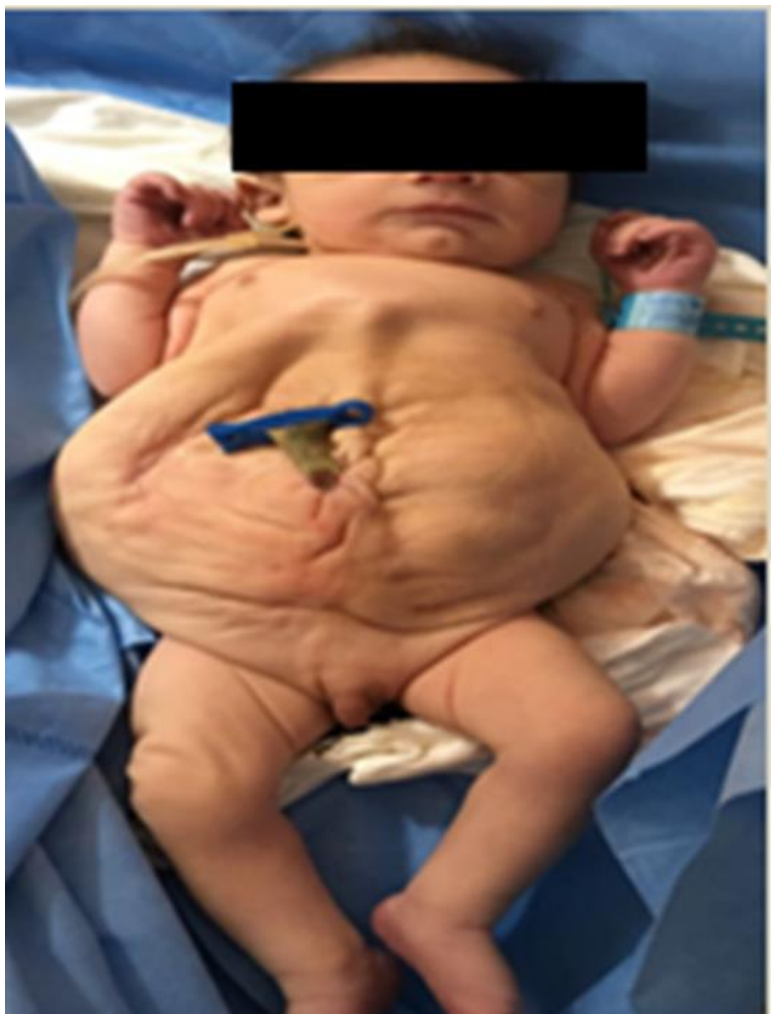

Figure 4. Aspect clinique d'un syndrome de Prune Belly à la naissance avec paroi abdominale flasque et cryptorchidie bilatérale 


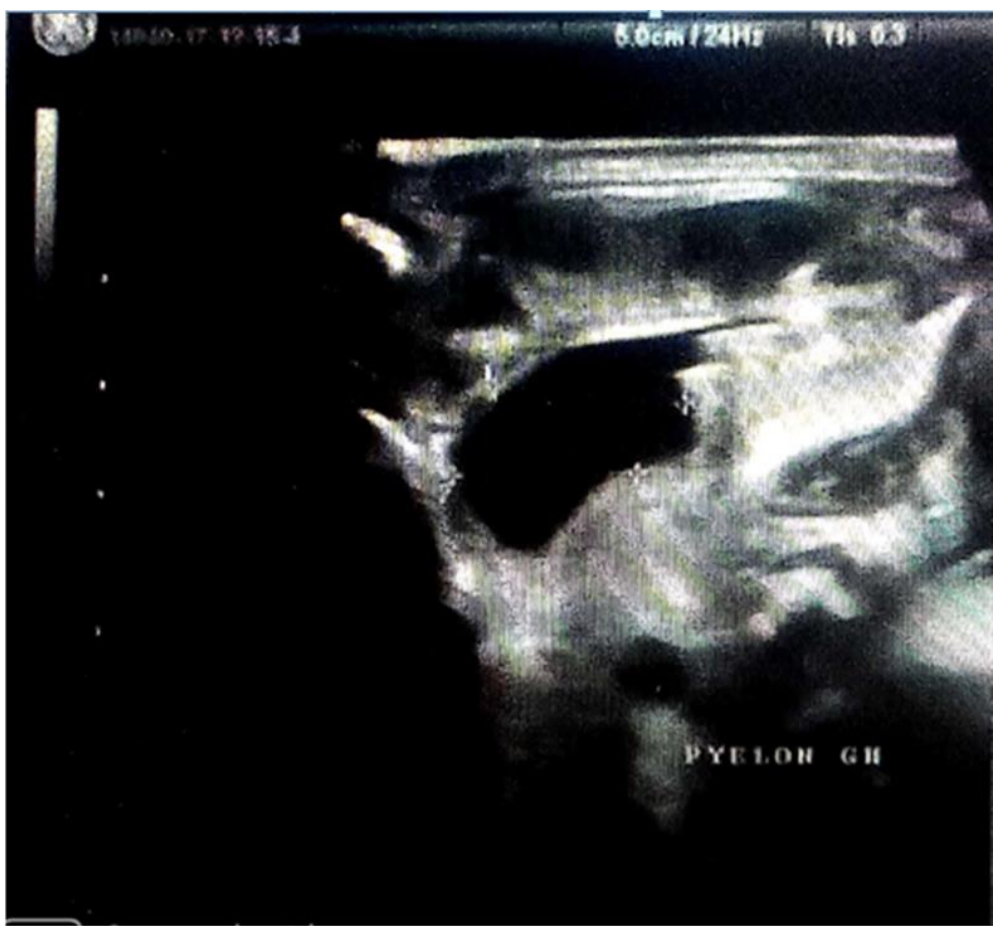

Figure 5. Echographie rénale post natale

Importante urétéro-hydronéphrose bilatérale avec mégavessie.

\section{Discussion}

Le syndrome de Prune Belly décrit pour la première fois par Frolich en 1839 est une affection rare, dont l'incidence est estimée à 1 cas sur 40000 naissances (Megan et al., 2006).C'est une malformation complexe et rare, caractérisée par la triade : aplasie ou grande hypoplasie de la musculature abdominale, des anomalies du tractus urinaire et cryptorchidie bilatérale. Cette affection est marquée par une nette prédominance masculine (plus de $95 \%$ des patients) et par la rareté des formes complètes chez la fille et c'est le cas de notre patient qui est de sexe masculin (Megan et al., 2006 ; Aaronson et al., 2001). Cette nette prédominance masculine a fait évoquer une possible participation génétique avec une transmission autosomique récessive liée au sexe (Ramasamy et al., 2005). Cependant, la présence de l'anomalie chez un seul des jumeaux dans deux cas de grossesse gémellaire monozygote rend ce mode de transmission plus difficile à élucider (Maugey-Laulom et al., 2000).Ce syndrome résulte de la défaillance de migration ou différenciation du mésoderme latéral en musculature abdominale et en musculature du tractus urinaire. L'uropathie correspond à une dysfonction ou à une obstruction de l'urètre provoquant une mégavessie et des méga-uretères (Driessen et al., 2014). Une hypoplasie de la prostate est fréquente chez les garçons. Chez les filles, cette séquence associe, à l'uropathie et à l'anomalie de la paroi 
abdominale, une atrésie vaginale, recto-vaginale, des fistules recto-vésicales ou un utérus bicorne (Ramasamy et al., 2005 ; Maugey-Laulom et al., 2000).

Le diagnostic prénatal repose sur l'échographie obstétricale capable de détecter les anomalies de l'appareil urinaire associées à l'aspect typique de la paroi abdominale; ainsi les signes échographiques sont: oligoamnios (fréquemment retrouvé : facteur de mauvais pronostic), dilatation majeure de la vessie occupant tout le contenu de l'abdomen fotal, dilatation pyélocalicielle unilatérale ou bilatérale, paroi abdominale amincie proéminente en coupes sagittales (Grahame et al., 2011). En post natal, le diagnostic repose essentiellement sur la clinique et l'échographie abdomino-pelvienne compétées par une TDM (Tomodensitométrie) abdomino-pelvienne, une échographie trans-thoracique à la recherche de malformation cardiaque, un bilan rénal pour évaluer la fonction rénale, une échographie des hanches avec radiographie du squelette à la recherche de malformations ainsi qu'un caryotype à la recherche de suppression sur le facteur nucléaire 1-beta (HNF1beta) (Sutton et al., 2000); (Darmon et al., 2002). Notre malade avait la forme complète du syndrome avec notamment tous les éléments de la triade diagnostiquée. Il a bénéficié en post natal d'une échographie abdominopelvienne objectivant une importante urétèro-hydro-néphrose bilatérale associée à une mégavessie et une aplasie des muscles abdominaux avec une cryptorchidie. Une échographie trans-thoracique ainsi qu'une radiographie du squelette ont été faites et elles n'ont pas objectivé d'anomalies. Cependant, jusqu'à $75 \%$ des patients présentant ce syndrome associent d'autres malformations, à savoir des malformations pulmonaires, cardiaques, squelettiques, gastro- intestinales et génitales. Ces malformations ont été rapportées avec une incidence de $25 \%$ pour les cardiovasculaires, $24 \%$ pour les gastro-intestinales, $23 \%$ pour les musculo-squelettiques ,58\% pour les respiratoires et $15 \%$ pour les génitales. Les malformations respiratoires retrouvées sont l'hypoplasie pulmonaire et la malformation adénomatoide kystique, lesquelles peuvent entraîner différents degrés d'insuffisance respiratoire, raison principale de mortalité néonatale (Routh et al., 2010 ; Fallat et al., 2003).

Le principal diagnostic différentiel inclut les uropathies basses obstructives causées par des valves de l'urètre postérieur, une hypertrophie du détrusor, une agénésie urétrale ou des membranes congénitales urétrales (Writters et al., 2001).

La prise en charge thérapeutique repose essentiellement sur la chirurgie: l'abdominoplastie, l'orchidopexie et la reconstruction des voies urinaires. Pour les patients atteints d'une légère dysplasie de la paroi abdominale, les postures sont acceptables et ne nécessitent pas d'abdominoplastie. Cependant, pour les cas sévères, le traitement chirurgical est discuté au cas par cas. Les pyélostomies, urétérostomies et cystostomies 
sont également entreprises pour shunter l'urine temporairement chez certains nourrissons instables qui ne peuvent pas tolérer l'acte chirurgical. Parfois la transplantation rénale est inévitable pour les patients présentant une insuffisance rénale. Quoi qu'il en soit, que le traitement chirurgical soit entrepris ou non, les patients nécessitent des soins médicaux multidisciplinaires permanents et un suivi rapproché (Bankole Sanni et al., 2000 ; Diao et al., 2008). Notre malade a bénéficié juste d'un sondage vésical vu la fistule de l'ouraque qui ramène les urines à l'extérieur avec suivi multidisciplinaire. Le pronostic de ce syndrome varie selon la gravité de l'hypoplasie pulmonaire et des anomalies des voies urinaires. L'hypoplasie pulmonaire est la principale cause de mortalité à la période néonatale. La gravité des anomalies des voies urinaires et la fonction rénale déterminent non seulement la mortalité, mais aussi le pronostic à long terme (Ph. PETIT et al., 2000).

\section{Conclusion}

La découverte précoce de mégavessie à l'échographie anténatale nécessite toujours la recherche des syndromes associés et notamment le syndrome de Prune Belly qui est une entité rare et dont la découverte nécessite un suivi particulier à la recherche d'autres malformations associées qui déterminent non seulement la mortalité, mais aussi le pronostic à long terme.

\section{References:}

1. Aaronson, I. \& Cremin, B. (2001). Prune Belly syndrome in young females. Urol Radiol; 1:151.

2. Bankole Sanni, R., Coulibaly Denoulet, D., \& Mobiot, ML. (2000). Le traitement du syndrome du ventre en pruneau (prune belly syndrome) au CHU de Treichville. Ann d'urol; (32) : 23 -27.

3. Darmon, JC., Janky, E., \& Couchy, B. (2002). Syndrome de prune belly à propos d'un cas de récurrence. Rev Fr gynecol obstét; 87(3) : $151-154$.

4. Diao B, et al. (2008). Syndrome de Prune Belly : aspects épidémiologiques, cliniques et thérapeutiques. Progrès en Urologie; 18: 470-474.

5. Driessen, M., Chéreau, E., Aubry, MC., Vibert-Guigue, C., Ruano, R., \& Dommergues, M. (2014). Anomalies urogénitales foetales. EMC Obstétrique/Gynécologie; 9(1) : 1-20.

6. Fallat, ME., Skoog, SJ., Belman, AB., Eng, G., \& Randolph, JG. (2003). The prune belly syndrome: a comprehensive approach to management. J Urol; 142:802-805. 
7. Grahame, H., Smith, H., \& Andrew John Holland (2011). Prune belly syndrome. Pediatric Surgery International * December 2011; 10.1007/s00383-011-3046-6.

8. Maugey-Laulom, B., Brun, M., Chateil, JF., \& Diard, F. (2000). Diagnostic anténatal des uropathies malformatives. Encycl Méd Chir (Editions Scientifiques et Médicales Elsevier SAS) Paris 9 p.

9. Megan, M., Bogart, MD., Holly, E., Arnold, MD., Kenneth, E., \& Greer, MD. (2006). Prune Belly syndrome in two children and review of the literature. Pediatr Dermatol; 23(4):342-5.

10. Petit, PH., Dacher, J.N., Faure, F., Guys, J.M., Bourliere, B., Portier, F., Le Dosseur, P., Panuel, M., Devred, PH. (2000). Exploration radiologique des troubles mictionnels chez l'enfant. F.Radiol; 39(3) : 216- 224.

11. Ramasamy, R., Haviland, M., Woodard, JR., \& Barone, JG. (2005). Patterns of inheritance in familial Prune Belly syndrome. Urology; 65(6): 1227.

12. Routh, JC., Huang, L., Retik, AB., \& Nelson, CP. (2010). Contemporary epidemiology and characterization of newborn males with prune belly syndrome. Urology; 76:44-48.

13. Writters, I., Theyskens, C., van Hoestenberghe, R., Sierath, P., Gyselaers, W., \& Fryns, JP. (2001). Prenatal dignosis of nonobstructive megacystis as part of the megacystis-microcolon-intestinal hypoperistalsis syndrome with favourablepotsnatal come. Prenat Diagn 2001; 21:701-6. 